It is precisely under these conditions (Sun at or near the horizon) that the polarization compass works best, and may eventually achieve its widest application. It is offered as an inexpensive, simple-to-build but highly accurate instrument for desperate fog-bound compass adjusters and others who have difficulty determining which way they are headed.

\title{
The Conception and Development of Weir's Diagram
}

\author{
Charles H. Cotter
}

Just a century ago, in 1876 , Patrick Weir, an officer of a vessel trading between London and Australia, conceived the idea of a diagram that might facilitate finding the Sun's true azimuth for the purpose of checking the magnetic compass. Some thirteen years later Captain Weir's Diagram was the subject of a paper communicated by Sir William Thomson (later Lord Kelvin) to the Royal Society of Edinburgh. In his paper ${ }^{1}$ Weir outlined the train of reasoning by which he succeeded in constructing a novel diagram which was described by Professor $P$. G. Tait' as 'a singularly elegant construction which, not only puts in a new and attractive light one of the most awkward of the problems of spherical trigonometry, but it practically gives in a single-page diagram the whole content of the two volumes of Burdwood's Azimuth Tables'. Tait also remarked that the method supplied an interesting graphical plane construction of a function of three independent variables.

As a result of Weir's original conception a complete diagram ${ }^{3}$ covering all latitudes from $0^{\circ}$ to $60^{\circ}$ and all hour angles at 4 min. $\left(1^{\circ}\right)$ intervals was professionally drawn and published by the well-known nautical publisher J. D. Potter of the Minories in London. The original published diagram was mounted on card and appended to it were Captain Weir's instructions 'simplified by Professor George Darwin and Sir William Thomson'.

Weir related that to simplify the problem of finding the Sun's true azimuth he conceived the idea of 'projecting the Sun's path on to the plane of the horizon'. The line of his subsequent reasoning proceeded thus : To an observer at the Earth's north (or south) pole the projection of the Sun's diurnal path onto the plane of the observer's horizon is a circle and that, no matter what its declination, the Sun's bearing is the same at any given time each day-only its altitude being affected by a change in declination. To an observer on the equator the projection of the Sun's daily path onto the plane of the observer's horizon, when the Sun's declination is $0^{\circ}$, is a straight line which joins the east and west points of the horizon. Also, to an observer on the equator, the rising and setting amplitudes and the Sun's meridian zenith distance are equal in magnitude and name to the Sun's declination.

Weir then posed the question : If the Sun's path is projected as a circle for an observer in lat. $90^{\circ}$, and as a straight line for an observer in lat. $0^{\circ}$, what is its 
projection for an observer in some intermediate latitude? He came to the conclusion that it must be an ellipse, arguing by analogy that while the crank-pin of a steam-engine describes a circle and the crosshead travels in a straight line, any intermediate point on the connecting-rod describes an ellipse. He deduced the relative lengths of the major and minor axes of the ellipse representing the Sun's path, for any given latitude, by considering the nature of the shadow cast by a disc when the plane of the disc, initially placed 'flat-on' to the light-source, is turned about a diameter through a right angle. If the disc is canted at an angle $\theta^{\circ}$ from being 'edge-on' to the light, the shadow will be elliptical in outline and such a shape corresponds with that of the Sun's path projected onto the plane of the horizon of an observer in lat $\theta^{\circ}$. He could see that whatever may be the obliquity of the disc, its shadow will be an ellipse of the same dimensions as that representing the Sun's daily path in the latitude corresponding to that obliquity. He also could see that the major axes of a family of ellipses, each ellipse corresponding to a particular latitude, would coincide at the east and west points of the horizon, and that the minor axes would be proportional in length to the sine of the angle of obliquity or the latitude of the observer.

The arrangement answered well when the Sun's declination is zero, but to allow for declination $\delta$ for an observer in latitude $\phi$, Weir showed that the position of the observer would have to be shifted in the opposite direction (south if the declination is north, and north if the declination is south) by an amount proportional to $\tan \delta \cos \phi$. But this he could see would require a separate scale of declination for each ellipse of latitude. To overcome this inconvenience Weir decided to vary the sizes of the ellipses instead of varying the scale of declination. Instead of multiplying $\tan \delta$ by $\cos \phi$ he divided the major and minor axes of each ellipse by $\cos \phi$ so that :

$$
\begin{aligned}
& \text { length of major axis }=R / \cos \phi=R \sec \phi \\
& \text { length of minor axis }=R \sin \phi / \cos \phi=R \tan \phi
\end{aligned}
$$

where $R$ is half the straight-line distance between the projections of the east and west points. In this way the relative lengths of the major and minor axes of the latitude ellipses, as demonstrated by the disc shadow, are preserved.

The arrangement allowed the declination to be measured or marked off on the scale of latitude. Moreover, the foci of all the latitude ellipses are located at the ends of the projection of the Sun's path for latitude $0^{\circ}$. 'This was a great consideration' Weir remarked 'when ellipses have to be drawn with pins and thread'.

Weir took for granted that the Sun's path projected onto the plane of the horizon is exactly the same at all times of the year, the whole ellipse representing the path shift to north or south (according as the declination is north or south) through a distance proportional to $\tan \delta$. He mentioned that it is impossible to make the ellipses shift on a diagram; and to overcome the difficulty he supposed the observer's position to be shifted through a corresponding distance in the opposite direction.

Having computed the dimensions of the latitude ellipses, the next problem was to fix the Sun's positions on them for different times of day. It was evident to Weir that the noon-line for all latitudes corresponds to the meridian, and therefore to the minor axes of the ellipses. He was equally certain that the 6 o'clock hour circle would be represented by a straight line at right angles to the meridian, so that it corresponds with the major axes of the ellipses. He determined the positions of the intermediate 'time lines' as follows: Taking any ellipse of 
latitude, with centre at a point midway between the foci of the ellipse and with radius equal to half the major axis of the ellipse, he described a circle. This circle was divided uniformly into time intervals. From these divisions he drew lines parallel to the meridian. The points at which these cut the ellipses denote positions of the Sun for different latitudes corresponding to the times denoted on the graduated circle. This routine was repeated for each of the ellipses and the points on the ellipses for each particular time were joined 'in a regular sweep'. Weir remarked that the resulting curves are 'very much like hyperbolae'. In fact they are hyperbolae but Weir could not prove this. To facilitate measuring azimuths a graduated circle was drawn centred at the intersection of the major and minor axes of the ellipses.

When Weir's diagram was first described in print in I 889 the two methods most commonly used for finding azimuths were a mechanical device called a 'spherograph', the invention of a Royal Naval engineer officer named Saxby, and Burdwood's Azimuth Tables. On grounds of expense Weir considered his diagram to be superior and his remarks are interesting:

The spherograph is rather a costly affair (about $£_{2}$ I believe), and for that reason has not come into general use. Burdwood's tables are not so expensive, but still they cost $12 \mathrm{~s}$. 6d. per set, whereas this diagram could be published in chart form at certainly not more than one-fifth of that price.

Following Weir's paper Professor Tait, at the request of Sir William Thomson who was unable to communicate Weir's paper personally, added a note on the principle of the method:

In the usual notation for spherical triangles, if $\mathrm{A}$ be the zenith, $\mathrm{C}$ the pole, and $\mathrm{B}$ a heavenly body (whose declination is $\delta$ ), $\mathrm{C}$ is the hour angle $(h), b$ the colatitude $(\pi / 2-\lambda)$, and $A$ the supplement of the azimuth. Hence, from the formula

$$
\cot a \sin b=\cot A \sin C+\cos b \cos C,
$$

we have at once:

$$
\tan (\mathrm{az})=\sin h /(\sin \lambda \cos h+\tan \delta \cos \lambda)
$$

Captain Weir, in his diagram, virtually puts

$$
\begin{aligned}
& x=\sin h \sec \lambda \\
& y=\cos h \tan \lambda
\end{aligned}
$$

so that

$$
\tan (a z)=x /(y-\tan \delta)
$$

$x$ and $y$ being found by the intersection of the confocal conics :

$$
\begin{gathered}
x^{2} / \sec ^{2} \lambda+y^{2} / \tan ^{2} \lambda=1 \text { (the latitude ellipse) } \\
x^{2} / \sin ^{2} h-y^{2} / \cos ^{2} h=1 \text { (the hour-angle hyperbola). }
\end{gathered}
$$

Captain Patrick Weir, a thorough-going practical seaman with very limited mathematical training, deserves credit for the ingenious way in which he reasoned out the construction of his cleverly-designed diagram. Praise is also due to the mathematicians Thomson, Tait and Darwin-each a philosophical giant-who gave Captain Weir their enthusiastic support and were doubtless instrumental in getting the diagram published, and into the hands of those who were able to put it to profitable use. 


\section{REFERENCES}

1 Weir, Patrick (1889). Theoretical Description of a 'New Azimuth Diagram.' Communicated by Sir W. Thomson. Proceedings of the Royal Society of Edinburgh (Session 1888-89). pp. 354-59.

2 Tait, P. G. (1889). Note on Capt. Weir's Paper. Proceedings of the Royal Society of Edinburgh (Session 1888-89). pp. 359-61.

3 Weir's Diagram was published in chart form by the British Admiralty in 1890 but was withdrawn in about 1965. A detailed account of its principles and use is given in The Admiralty Manual of Navigation (1938) Vol. III.

\section{Record}

\section{XIth INTERNATIONAL HYDROGRAPHIC CONFERENCE}

\section{Monaco I8-30 April 1977}

The following report by $\mathrm{Dr}$. Charles $\mathrm{H}$. Cotter is followed by the text of his address to the conference on the project sponsored by IAIN to compile an annotated bibliography of astronomical navigation tables and methods.

THE number of Member States of the International Hydrographic Organization (IHO) is now 47; this includes the U.S.S.R. which joined the Organization in February, 1977. The broad aims of the IHO, which is an intergovernmental organization, are to coordinate the work of national hydrographic offices in surveying and charting the seas, and to reduce duplication of effort in these activities. IHO cooperates with several international and national bodies, including the United Nations Conference on the Law of the Sea; the International Oceanographic Commission (IOC) of UNESCO; and IMCO; IALA; WMO; and the Scientific Committee on Oceanic Research (SCOR).

The official opening of the eleventh International Hydrographic Conference, at which IAIN was represented by M. W. Richey, its Secretary General, Professor P. Hugon, President of the French Institute of Navigation, and Captain C. H. Cotter, took place on 18 April 1977, the ceremony having been conducted by His Excellency M. André Saint-Mieux, Minister of State, Principality of Monaco. The President of the Directing Committee of the International Hydrographic Bureau (IHB), Rear Admiral G. S. Ritchie, R.N. (Ret.), then presented his report in which he reviewed the progress of the IHB and focussed attention on problems facing IHO.

The programme of work undertaken by the IHO is formulated at its quinquennial conferences, at which reports of progress since the preceding conference are presented to the conference delegates. Four commissions and two working groups were established at the tenth Conference held in 1972. The 